\title{
Radiation proctitis: predictors of mortality and inpatient outcomes in the United States
}

\author{
Dushyant S. Dahiyaa, Asim Kichloo a, Abhilash Perisettib, Hafeez Shakac, Jagmeet Singh ${ }^{d}$, \\ Sumant Inamdare
}

Central Michigan University College of Medicine, Saginaw, MI; Parkview Cancer Institute, Fort Wayne, IN; John H. Stroger, Jr. Hospital of Cook Country, Chicago, IL; Guthrie Robert Packer Hospital, Sayre, PA; University of Arkansas for Medical Sciences, Little Rock, AR, USA

\begin{abstract}
Background We aimed to evaluate the characteristics of hospitalizations for radiation proctitis (RP).

Methods The National Inpatient Sample (NIS) was analyzed to identify RP hospitalizations for 2016 and 2017. Outcomes included mortality, predictors of mortality, mean length of stay (LOS), mean total hospital cost (THC), and numerous system-based complications.

Results We identified 16,810 adult hospitalizations for RP. On admission, an initial diagnosis of RP was established for only $27.54 \%$ of these patients. The mean age was $72.3 \pm 0.5$ years and $30.2 \%$ of the study population was female. Whites made up $68.7 \%$ of the study population. Most hospitalizations for RP were at large (51\%), urban (93.6\%), and teaching (71.1\%) hospitals. The inpatient mortality for RP hospitalizations was $1.7 \%$. After adjusting for biodemographic factors, hospitalization characteristics and comorbidities, older age and protein energy malnutrition (PEM) were associated with higher odds of inpatient mortality. The mean LOS and THC for RP hospitalizations were 5.6 days and $\$ 53,800$, respectively. Inpatient complications associated with RP included acute renal failure (19.7\%), sepsis (4.4\%), deep vein thrombosis (3.7\%), acute respiratory failure $(3.3 \%)$, and pulmonary embolism $(0.7 \%)$.
\end{abstract}

Conclusion Inpatient mortality for RP was $1.7 \%$. Older age and PEM were associated with higher odds of inpatient mortality.

Keywords Proctitis, radiation, predictors, outcomes, mortality

Ann Gastroenterol 2021; 34 (1): 1-5

\section{Introduction}

The use of ionizing radiation, alone or in combination with other therapies, has become an essential component of the treatment regimens available for cancers worldwide [1].

${ }^{a}$ Central Michigan University College of Medicine, Saginaw, MI

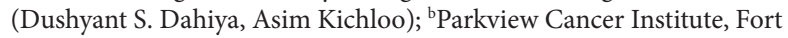
Wayne, IN (Abhilash Perisetti); 'John H. Stroger, Jr. Hospital of Cook County, Chicago, IL (Hafeez Shaka); dGuthrie Robert Packer Hospital, Sayre, PA (Jagmeet Singh); ' ${ }^{-}$niversity of Arkansas for Medical Sciences, Little Rock, AR (Sumant Inamdar), USA

\section{Conflict of Interest: None}

Correspondence to: Dushyant Singh Dahiya, MD, Central Michigan University College of Medicine, Saginaw, MI, USA, 1000 Houghton Ave, Saginaw, MI, USA 48603, e-mail: dush.dahiya@gmail.com

Received 24 September 2021; accepted 21 November 2021; published online 11 December 2021

DOI: https://doi.org/10.20524/aog.2021.0684
Although, the goal of radiation therapy (RT) is to target and damage rapidly dividing cancer cells, normal tissues may also be affected, often leading to complications and/or toxicities $[2,3]$. One of the most frequently encountered complications of RT is radiation proctitis (RP), characterized by damage to the rectal epithelium secondary to the use of ionizing radiation $[2,4]$. $\mathrm{RP}$ can be classified as acute (ARP) or chronic (CRP), based on the amount of time between the initiation of RT and the development of clinical symptoms. ARP is often selflimiting; however, CRP is usually more severe and requires intervention [5-8]. Because of the significant variability in reporting of RP, its exact incidence and prevalence rates in the general population are currently unknown. Additionally, there is a significant paucity of data on adverse outcomes, such as inpatient mortality, common complications and the burden of the disease on the United States (US) healthcare system, even though a sizable proportion of the population in the US is at increased risk of developing RP. Hence, this study was designed to outline the spectrum of clinical presentations and biodemographic characteristics of RP in an inpatient setting. 
In addition, we detail the extent of the disease burden of the US healthcare system in terms of length of hospitalization and mean healthcare costs, and also focus on the adverse outcomes, predictors of mortality and complications associated with RP hospitalizations.

\section{Materials and methods}

\section{Design and data source}

This was a retrospective study design that used the National Inpatient Sample (NIS) database to evaluate all adult ( $\geq 18$ years) hospitalizations for RP in the US between January 1, 2016, and December 31, 2017. The NIS belongs to the group of Healthcare Cost and Utilization Project (HCUP) databases, which collect inpatient billing data from community hospitals across the US, covering more than $95 \%$ of the US population [9]. The database approximates a $20 \%$ stratified sample and is weighted to obtain national estimates for analysis [10]. For the 2016 and 2017 study period, it was coded using the International Classification of Diseases (ICD)-9 and ICD-10 coding systems.

\section{Study population}

The study population included all adult ( $\geq 18$ years) hospitalizations with a diagnosis of RP (K62.7). Individuals $<18$ years of age were excluded from the study.

\section{Outcome measures}

The top 10 principal diagnoses at admission and their proportions among hospitalizations with RP were identified. The outcomes included biodemographic characteristics, inpatient mortality, mean length of stay (LOS), mean total hospital cost (THC), and system-based complications such as acute respiratory failure, acute renal failure, sepsis, deep vein thrombosis and pulmonary embolism. We also identified independent predictors of inpatient mortality for RP hospitalizations.

\section{Statistical analysis}

Stata Version 16 software (StataCorp, Texas, USA) was used for the statistical analysis. All analyses included in the study were for weighted samples to obtain national estimates. HCUP rules and regulations were thoroughly followed in utilizing the NIS database. Independent predictors of inpatient mortality were identified using multivariate logistic regression analysis following a univariate screen. P-values $<0.05$ were considered statistically significant. In addition, we report no missing data for the variables and outcomes analysed.

\section{Ethical considerations}

The NIS database lacks patient identifiers. Hence, this this study was exempt from Institutional Review Board (IRB) approval as per guidelines put forth by our institutional IRB for analysis of inpatient databases.

\section{Data availability statement}

The NIS database is publicly available at https://www.hcupus.ahrq.gov.

\section{Results}

\section{Admission diagnoses for radiation proctitis hospitalizations}

At the time of admission, an initial diagnosis of RP was established for $27.54 \%$ of the patients (Table 1). Other common diagnoses at initial presentation included gastrointestinal hemorrhage $(4.13 \%)$, sepsis secondary to an unspecified cause (3.75\%), hemorrhage of the anus and rectum (2.74\%), melena (2.47\%), angiodysplasia of the colon with hemorrhage $(2.17 \%)$, bleeding diverticulosis (1.93\%), acute renal failure (1.90\%), radiation-induced gastroenteritis (1.52\%), and irradiation cystitis with hematuria (1.37\%) (Table 1).

Table 1 Top 10 initial diagnoses at the time of admission for patients with radiation proctitis

\begin{tabular}{lcc}
\hline Initial diagnosis & $\begin{array}{c}\text { Total number of } \\
\text { hospitalizations }\end{array}$ & Proportion (\%) \\
\hline Radiation proctitis & 4630 & 27.54 \\
$\begin{array}{l}\text { Gastrointestinal } \\
\text { hemorrhage (unspecified) }\end{array}$ & 695 & 4.13 \\
$\begin{array}{l}\text { Sepsis (unspecified) } \\
\begin{array}{l}\text { Hemorrhage of the anus } \\
\text { and rectum }\end{array}\end{array}$ & 630 & 3.75 \\
$\begin{array}{l}\text { Melena } \\
\text { Angiodysplasia of the } \\
\text { colon with hemorrhage }\end{array}$ & 460 & 2.74 \\
$\begin{array}{l}\text { Diverticulosis with } \\
\text { bleeding }\end{array}$ & 365 & 2.47 \\
$\begin{array}{l}\text { Acute renal failure } \\
\text { Radiation-induced } \\
\text { gastroenteritis }\end{array}$ & 320 & 2.17 \\
$\begin{array}{l}\text { Irradiation cystitis with } \\
\text { hematuria }\end{array}$ & 255 & 1.93 \\
\hline
\end{tabular}




\section{Hospitalization characteristics for RP}

We identified 16,810 hospitalizations for RP during 2016-2017 (Table 2). The mean age was noted to be $72.3 \pm 0.5$ years. Females made up $30.2 \%$ of all RP hospitalizations. Whites $(68.7 \%)$ made up the majority of the cohort, followed by Blacks (15.7\%), Hispanics (7.6\%) and other races (8\%). Additionally, most RP hospitalizations were reported at large (51\%), urban $(93.6 \%)$, and teaching (71.1\%) hospitals. From a payment perspective, Medicare was found to be the largest insurer $(77.2 \%)$, followed by private insurance (15.3\%) and Medicaid (6.5\%).

\section{Outcome measures}

The inpatient mortality for RP hospitalizations was found to be $1.7 \%$ (Table 3 ). Additionally. the mean LOS was 5.6 days (95\% confidence interval $[\mathrm{CI}]$ 5.4-5.8; $\mathrm{P}<0.001$ ), and the mean THC was $\$ 53,800$ (95\%CI 51,100-56,500; P<0.001). Systembased complications associated with RP hospitalizations included acute renal failure (19.7\%), sepsis (4.4\%), deep vein thrombosis $(3.7 \%)$, acute respiratory failure $(3.3 \%)$, and pulmonary embolism $(0.7 \%)$.

\section{Predictors of inpatient mortality}

After a multivariate regression analysis adjusting for biodemographic factors, hospitalization characteristics and numerous comorbidities, older age (adjusted odds ratio [aOR] 1.03, 95\%CI 1.01-1.05; $\mathrm{P}=0.033)$ and protein energy malnutrition (PEM) (aOR 4.87, 95\%CI 2.76-8.59; $\mathrm{P}<0.001$ ) were found to be associated with increased odds of inpatient mortality among RP hospitalizations (Table 4).

\section{Discussion}

The present study used the largest inpatient database to evaluate the clinical presentation and burden of RP on the US healthcare system. This is the only study that we are aware of that provides data on RP hospitalizations. In modern day medicine, RT, alone or in combination with other treatment modalities, has become an essential component in the treatment regimens of cancers [1]. However, data on RP in an inpatient setting are lacking. As the use of RT continues to increase, physicians are more likely to encounter patients with RP, despite the availability of progressively less toxic radiotherapy techniques [11]. Therefore, it becomes essential to identify hospitalization characteristics and the disease burden on the US healthcare system.

In this study, of the 16,810 adult RP hospitalizations, only $27.54 \%$ were identified to be RP at initial presentation. All other RP hospitalizations had a different admitting diagnosis (Table 1). This may be due to a significant symptomatic
Table 2 Biodemographic and hospitalization characteristics of patients suffering from radiation proctitis

\begin{tabular}{|c|c|}
\hline Hospitalization characteristics & Value \\
\hline Total hospitalizations & 16,810 \\
\hline Mean age (years) \pm standard error & $72.3 \pm 0.5$ \\
\hline $\begin{array}{l}\text { Sex } \\
\text { Female } \\
\text { Male }\end{array}$ & $\begin{array}{l}30.2 \\
69.8\end{array}$ \\
\hline $\begin{array}{l}\text { Racial distribution } \\
\text { White } \\
\text { Black } \\
\text { Hispanic } \\
\text { Other }\end{array}$ & $\begin{array}{r}68.7 \\
15.7 \\
7.6 \\
8.0\end{array}$ \\
\hline $\begin{array}{l}\text { Charlson comorbidity index score } \\
0 \\
1 \\
2 \\
\geq 3\end{array}$ & $\begin{array}{l}13.7 \\
13.4 \\
17.5 \\
55.4\end{array}$ \\
\hline $\begin{array}{l}\text { Insurance type } \\
\text { Medicaid } \\
\text { Medicare } \\
\text { Private } \\
\text { Uninsured }\end{array}$ & $\begin{array}{c}77.2 \\
6.5 \\
15.3 \\
1.0\end{array}$ \\
\hline $\begin{array}{l}\text { Comorbidities } \\
\text { Hypertension } \\
\text { Congestive heart failure } \\
\text { Diabetes mellitus } \\
\text { Smoking } \\
\text { Dyslipidemia } \\
\text { Chronic obstructive pulmonary disease } \\
\text { Chronic ischemic heart disease } \\
\text { Obesity } \\
\text { Chronic kidney disease } \\
\text { Protein energy malnutrition } \\
\text { Anemia } \\
\text { Prior cerebrovascular accident } \\
\text { Oxygen dependence }\end{array}$ & $\begin{array}{l}41.4 \\
20.4 \\
31.2 \\
37.8 \\
43.6 \\
15.9 \\
33.6 \\
11.0 \\
23.4 \\
11.4 \\
44.0 \\
2.8 \\
2.3\end{array}$ \\
\hline $\begin{array}{l}\text { Hospital characteristics } \\
\text { Small sized hospitals } \\
\text { Medium sized hospitals } \\
\text { Large sized hospitals } \\
\text { Teaching status }\end{array}$ & $\begin{array}{l}18.9 \\
30.1 \\
51.0 \\
71.1\end{array}$ \\
\hline
\end{tabular}

Numbers are percentages unless otherwise stated

overlap with other disease processes or admissions due to complications of RP. Additionally, as per the literature, patients $\leq 60$ years of age were noted to have a greater risk of $\mathrm{RP}$ compared to those $>60$ years old; however, it is unclear whether these patients have an increased radiation-induced inflammatory response, or whether this is due to the fact that they are more likely to report symptoms compared to an older demographic [12]. Even though this might be true, our study indicates that hospitalizations due to RP were more common in the older patients (mean age $72.3 \pm 0.5$ years), with a male predominance. Moreover, racial differences were also noted for RP hospitalizations, as Whites made up much of the cohort, followed by Blacks, Hispanics, and other races. This may, in part, be secondary to a higher prevalence of specific cancers, 
Table 3 Inpatient outcomes for radiation proctitis hospitalizations

\begin{tabular}{lc}
\hline Outcomes & Proportion $(\mathrm{N}=16,810)$ \\
\hline Inpatient mortality (\%) & 1.7 \\
Length of stay (mean days) & 5.6 \\
Mean total hospital charges (USD) & 53,800 \\
Acute renal failure (\%) & 19.7 \\
Sepsis (\%) & 4.4 \\
Deep vein thrombosis (\%) & 3.7 \\
Acute respiratory failure (\%) & 3.3 \\
Pulmonary embolism (\%) & 0.7 \\
\hline
\end{tabular}

Table 4 Predictors of inpatient mortality for radiation proctitis hospitalizations

\begin{tabular}{lccc}
\hline Predictors & $\begin{array}{c}\text { Adjusted } \\
\text { odds ratio }\end{array}$ & $\begin{array}{c}95 \% \text { Confidence } \\
\text { interval }\end{array}$ & P-value \\
\hline Age & 1.03 & $1.01-1.05$ & 0.033 \\
Hospital location & 0.50 & $0.22-1.14$ & 0.100 \\
$\begin{array}{l}\text { Congestive heart } \\
\text { failure }\end{array}$ & 1.58 & $0.91-2.77$ & 0.107 \\
$\begin{array}{l}\text { Chronic liver } \\
\text { disease }\end{array}$ & 1.72 & $0.80-3.68$ & 0.165 \\
$\begin{array}{l}\text { Protein energy } \\
\text { malnutrition }\end{array}$ & 4.87 & $2.76-8.59$ & $<0.001$ \\
\begin{tabular}{l} 
Dyslipidemia \\
\hline
\end{tabular} & 0.50 & $0.29-0.88$ & 0.017 \\
\hline
\end{tabular}

requiring higher utilization of RT for Whites compared to other races [13]. Furthermore, we also noted a higher proportion of chronic medical conditions, such as hypertension, diabetes mellitus and dyslipidemia, and a history of smoking in these patients (Table 1). These findings were expected, as studies have reported that the presence of chronic medical conditions may increase tissue injury and hinder tissue repair post RT [4].

The prognosis of RP depends on the severity and extent of radiation-mediated damage. ARP is often self-limiting with minimal complications but in cases of severe RP, a decrease in the health-related quality of life has been observed in $30 \%$ of the patients [14]. However, there are no data on mortality for RP hospitalizations. In this study, the inpatient mortality for RP hospitalizations was 1.7\% for 2016 and 2017 (Table 3). After a multivariate regression analysis adjusting for biodemographic factors, hospitalization characteristics and comorbidities, we observed that older age and PEM were associated with higher odds of inpatient mortality (Table 3). This may be because elderly patients have poor wound healing secondary to decreased synthesis of extracellular matrix (ECM), age-associated microcirculation changes leading to a higher likelihood of hypoperfusion to tissues, and a significant number of comorbid medical conditions leading to increased complications and adverse mortality outcomes of RP [15]. Additionally, the greater odds of mortality in patients with PEM may be due to the decreased availability of substrate required for the generation of ECM components for tissue repair [16,17]. Lack of adequate tissue repair may lead to higher rates of complications and deaths. This is of particular relevance when we consider that some preliminary clinical experiences have proved that a commonly PEM-related physical condition, namely sarcopenia, does not negatively affect overall survival among patients irradiated for some pelvic malignancies [18]. This result is probably due to the fact that such clinical experiences are greatly underpowered to show a higher risk of death associated with RP among sarcopenic patients. This large population study has the merit of alerting radiation oncologists to such an issue. Furthermore, RP places a significant burden on the healthcare system, as the mean LOS was 5.6 days and the inpatient cost of management per patient was found to be $\$ 53,800$ for the study period.

From a hospital perspective, most RP hospitalizations were at large urban teaching hospitals, rather than small or medium sized non-teaching rural hospitals (Table 2). This could be attributed to fact that these large academic hospitals are usually tertiary care referral centres for large geographical regions. As such, they are fully equipped with experienced colorectal surgeons or gastroenterologists for treatment interventions, as the friable mucosa is highly prone to perforation, and have the resources to handle complex cases and presentations. Additionally, an urban location with a large population density is more likely to yield higher hospitalization rates compared to rural areas.

This study has several strengths, one of which is the large sample size. The study population was obtained from one of the largest inpatient databases available in the US. As the NIS database covers more than $95 \%$ of the US population, the outcomes and conclusions of this study are applicable to almost all inpatient admissions in the US. Moreover, through the unique study design, we focus on multiple outcomes, allowing for a detailed analysis.

However, we do acknowledge the limitations of our study. The NIS database does not contain data on the exact symptoms reported by patients at presentation, the severity of the disease at presentation, the time of diagnosis, the hospital course or the treatment aspects of RP. Additionally, as this was a retrospective study design, we acknowledge all biases associated with retrospective studies. Furthermore, the NIS database collects information about inpatient diagnosis from billing data rather than individual patients. Therefore, patients admitted on numerous occasions for the same diagnosis may have been included multiple times in the study cohort. Lastly, it should be noted that the NIS database stores information in the form of ICD codes, which are prone to coding errors. Despite these limitations, the large study cohort, unique methodology and analysis technique add useful information to the scarce literature on RP.

In conclusion, as the utilization of RT continues to rise for the management of cancers, physicians are likely to encounter increasing numbers of cases of RP. In this study, from 20162017 there were 16,810 RP hospitalizations with RP identified as the admitting diagnosis for only $27.54 \%$ of the patients. The mean age was noted to be 72.3 years. A male and White race predominance was also seen for the study period. Additionally, large urban teaching hospitals had the highest proportion of 
RP hospitalizations. Furthermore, inpatient mortality for RP hospitalizations was noted to be $1.7 \%$. After a multivariate regression analysis, older age and PEM were associated with higher risk of inpatient mortality. The mean LOS and THC were 5.6 days and $\$ 53,800$, respectively. Inpatient complications of $\mathrm{RP}$ included acute renal failure, sepsis, deep vein thrombosis, acute respiratory failure, and pulmonary embolism.

\section{Summary Box}

\section{What is already known:}

- Radiation proctitis (RP) is a frequent complication of pelvic radiation therapy

- Acute RP is often self-limiting; however, chronic $\mathrm{RP}$ is more severe and requires intervention

- $\mathrm{RP}$ is associated with poor quality of life and increased healthcare costs

\section{What the new findings are:}

- At the time of admission, an initial diagnosis of RP was established for only $27.54 \%$ of the patients

- Inpatient mortality for RP hospitalizations was noted to be $1.7 \%$

- Older age and protein-energy malnutrition were associated with a higher risk of inpatient mortality from RP

\section{References}

1. Jonathan D, Tward JD, Christopher J, et al. Radiation therapy and skin cancer. In: Natanasabapathi G. Modern practices in radiation therapy. InTeched 2012: pp. 207-246.

2. Kennedy GD, Heise CP. Radiation colitis and proctitis. Clin Colon Rectal Surg 2007;20:64-72.

3. Ferini G, Pergolizzi S. A ten-year-long update on radiation proctitis among prostate cancer patients treated with curative external beam radiotherapy. In Vivo 2021;35:1379-1391.

4. Do NL, Nagle D, Poylin VY. Radiation proctitis: current strategies in management. Gastroenterol Res Pract 2011;2011:917941.
5. Grodsky MB, Sidani SM. Radiation proctopathy. Clin Colon Rectal Surg 2015;28:103-111.

6. Denton A, Forbes A, Andreyev J, Maher EJ. Non surgical interventions for late radiation proctitis in patients who have received radical radiotherapy to the pelvis. Cochrane Database Syst Rev 2002; 1:CD003455.

7. Gilinsky NH, Burns DG, Barbezat GO, Levin W, Myers HS, Marks IN. The natural history of radiation-induced proctosigmoiditis: an analysis of 88 patients. QJMed 1983;52:40-53.

8. Lucarotti ME, Mountford RA, Bartolo DC. Surgical management of intestinal radiation injury. Dis Colon Rectum 1991;34:865-869.

9. Healthcare cost and utilization project. Introduction to the HCUP National Inpatient Sample (NIS) [Internet]. The National (nationwide) Inpatient Sample database documentation. Rockville, MD: Agency for Healthcare Research and Quality. Available from: https://www.hcup-us.ahrq.gov/db/nation/nis/NIS Introduction_2016.jsp and https://www.hcup-us.ahrq.gov/db/ nation/nis/NIS_Introduction_2017.jsp [Accessed 30 November 2021].

10. Houchens R, Ross, D, Elixhauser A, Jiang J. Nationwide Inpatient Sample (NIS) Redesign Final Report [Internet]. 2014. HCUP Methods Series Report \# 2014-04 ONLINE. April 4, 2014. U.S. Agency for Healthcare Research and Quality. Available from: http://www.hcup-us.ahrq.gov/reports/methods/methods.jsp [Accessed 30 November 2021].

11. Ferini G, Tripoli A, Molino L, et al. How much daily image-guided volumetric modulated arc therapy is useful for proctitis prevention with respect to static intensity modulated radiotherapy supported by topical medications among localized prostate cancer patients? Anticancer Res 2021;41:2101-2110.

12. Yang TJ, Oh JH, Son $\mathrm{CH}$, et al. Predictors of acute gastrointestinal toxicity during pelvic chemoradiotherapy in patients with rectal cancer. Gastrointest Cancer Res 2013;6:129-136.

13. Joh DY, Chen LN, Porter G, et al. Proctitis following stereotactic body radiation therapy for prostate cancer. Radiat Oncol 2014;9:277.

14. Lev EL, Eller LS, Gejerman G, et al. Quality of life of men treated with brachytherapies for prostate cancer. Health Qual Life Outcomes 2004;2:28.

15. Gould L, Abadir P, Brem H, et al. Chronic wound repair and healing in older adults: current status and future research. $J \mathrm{Am}$ Geriatr Soc 2015;63:427-438.

16. Stechmiller JK. Understanding the role of nutrition and wound healing. Nutr Clin Pract 2010;25:61-68.

17. Trujillo EB. Effects of nutritional status on wound healing. J Vasc Nurs 1993;11:12-18.

18. Ferini G, Cacciola A, Parisi S, et al. Curative radiotherapy in elderly patients with muscle invasive bladder cancer: the prognostic role of sarcopenia. In Vivo 2021;35:571-578. 